\title{
结合动态滑翔技术的小型太阳能 无人机飞行能量变化分析
}

\author{
刘思奇，白俊强
}

(西北工业大学 航空学院, 陕西 西安 710072)

\begin{abstract}
摘 要: 小型无人机由于其自身携能能力限制, 滞空时间较短, 因此通过应用太阳能技术和动态滑翔 技术获取外界能量对提升小型无人机续航性能意义重大。结合动态滑翔与太阳能各自的优势, 从小 型无人机能量交换角度入手, 分析了采用太阳能技术与动态滑翔技术的飞行器飞行过程中的能量变 化原理,推导了结合 2 种获能技术的最大能量获取策略。建立了基于梯度风场的小型无人机飞行动 力学模型, 并将其与太阳能动力能源系统模型相结合, 选择太阳能供应相对紧缺的黄昏时刻的环绕飞 行轨迹为目标航迹, 利用建立的模型进行了仿真验证, 验证了结合动态滑翔技术的小型太阳能无人机 相对于传统的太阳能飞行器盘旋飞行与纯动态滑翔飞行在能量获取上的优势, 证明了结合 2 种技术 对小型无人机续航能力提升的重要意义,为今后进一步研究全天候不间断飞行提供了基础。
\end{abstract}

\section{关 键 词: 动态滑翔; 小型无人机; 航迹优化; 太阳能; 能量分析}

中图分类号: V211.3

文献标志码: A

随着技术的不断发展, 小型无人机在军事与民 用领域应用日渐广泛, 但是小型无人机由于其自身 固有的尺寸限制致使在执行任务时所能携带的燃料 较少, 滞空时间较短, 限制了小型无人机的应用。因 此, 研究人员将目光转向了从飞行器之外获取额外 能量的方法, 太阳能动力驱动与动态滑翔技术就是 当前研究较为热门的能够使飞行器从外界获得额外 能量进而有效提升航时与航程的技术手段。

太阳能动力驱动技术以太阳辐射作为能量来 源。因其能源形式的特殊性, 太阳能飞机可以通过 光电转化实现能量补充, 与现有常规飞行器相比具 有明显优势, 是超长航时飞行器的重要发展方向。 太阳能飞机在持久监视、预警侦察、数据中继、边界 巡逻等领域拥有广阔的应用前景, 是各军事科技强 国研究的重点。但是, 小型无人机由于自身尺寸限 制与近地面的工作环境, 无法搭载大面积的太阳能 光电转化装置与储能装置, 单纯依靠太阳能技术对 其航时与航程的提高有限。为进一步提高小型无人 机的滞空性能, 在使用太阳能技术的同时引人动态
文章编号 : 1000-2758(2020)01-0048-10

滑翔技术进一步提高外界能量吸收效率。

动态滑翔技术是一种新兴的适用于小型无人机 的增程技术, 在不改变原有飞行器外形参数的条件 下, 仅通过改变飞行控制策略即能有效吸收外界横 向梯度风场能量, 减少小型无人机飞行能量消耗, 是 现在国际上研究的前沿技术之一。自然界中漂泊信 天翁是动态滑翔应用的典型示例, Sachs 等人通过实 验得出信天翁能够以 $81 \mathrm{~W}$ 飞行功率, 在 13.2 天内 不间断地从南佐治亚飞往西南太平洋, 消耗相当于 $5.6 \sim 11.9 \mathrm{~L}$ 汽油的能量, 远超其自身能提供的能 量 ${ }^{[1]}$ 。动态滑翔技术的优势在于仅通过飞行控制 和航迹规划修改飞行策略即可实现从梯度风场中获 取能量, 和太阳能技术相结合后不会影响太阳能动 力系统的布局和使用, 通过优化其飞行姿态控制, 还 可以使太阳能获取能力得到增强。

近年来, 太阳能技术与动态滑翔技术都取得了 新的发展。动态滑翔方面, Zhao 利用 NPSOL 软件 模拟了动态滑翔飞行器的最小燃油消耗 ${ }^{[2]}$, Sachs 等则研究了动态滑翔所需的最小风切变 ${ }^{[3]}$ 。 
Deittert 等利用微分平滑方法对小型无人机动态滑 翔飞行路径进行了研究 ${ }^{[4]}$ 。国内近年对于动态滑 翔的研究也取得了一定的成果, 朱炳杰等利用高斯 伪谱法分析了动态滑翔最优航迹中的能量变化 ${ }^{[5]}$ 。 刘多能等利用 Runge-Kutta 积分方法针对不同类型 的环绕形态及迁移形态的动态滑翔飞行轨迹进行了 优化求解 ${ }^{[6]}$ 。单上求等对动态滑翔飞行过程中的 能量转化机理进行了研究 ${ }^{[7]}$ 。太阳能方面, 美国密 歇根大学的 Spangelo 等推导了太阳能飞行器的飞行 动力学模型 ${ }^{[8]}$; 古克兰的 Sineglazov 等采用 Noth 提 出的总体设计方法, 进行了太阳能飞行器能源系统 的设计 ${ }^{[9-10]}$ 。昌敏以翼载荷为研究对象, 对太阳能 总体设计中的关键参量的进行了敏感度分析, 并提 出了以能量为中心的太阳能飞机初始方案设计方 法 $^{[11-12]}$ 。马东立基于重力储能原理建立了变高度 轨迹各阶段及其时间节点的物理数学模型, 研究了 相关总体技术参数对变高度轨迹应用效果的 影响 ${ }^{[13]}$ 。

目前关于小型无人机太阳能技术和动态滑翔技 术的研究大都局限于各自技术本身, 受小型无人机 尺寸与携能-载荷矛盾影响, 2 种技术在应用过程中 都各自具有其局限性:太阳能无人机增加携带太阳 能电池板和蓄能电池与携带载荷重量矛盾, 而动态 滑翔技术从风场中吸收的富余能量不易咜存, 同时 要求保持周期性飞行。将 2 种技术相结合, 同时发 挥两者的优势从外界获取能量, 动态滑翔保持周期 飞行从风场中获取能量, 维持飞行器飞行状态过程 中太阳能系统可以捾存更多能量, 以提升滞空能力, 进一步提升小型无人机的航程与航时。

本文从能量交换角度人手, 分析了采用太阳能 技术与动态滑翔技术的飞行器飞行过程中的能量变 化原理, 推导了结合 2 种获能技术的最大能量获取 策略; 建立了基于梯度风场的小型无人机飞行动力 学模型, 并将其与太阳能动力能源系统模型相结合, 使之能够较为准确地反映同时使用太阳能技术与动 态滑翔技术飞行器的飞行特征与能量变化规律。利 用能量分析得到了综合应用 2 种技术的飞行器最佳 获能策略, 通过对比太阳能飞行器传统盘旋、纯动态 滑翔飞行的飞行轨迹, 对其能量收支变化进行了深 人分析研究, 证明了结合 2 种技术能够为小型无人 机带来更大的能量优势, 对提高小型无人机的续航 性能具有十分重要的意义, 为今后研究全天候不间 断飞行提供了基础。

\section{1 建模分析}

\section{1 太阳能动力能源系统建模}

太阳能飞机通过光伏电池实现光电转化, 将太 阳能转化为电能, 再通过电动机将电力转化为飞行 器的机械能。当获取的太阳能大于飞行所必须的能 量消耗时, 可以将其以电能的形式它存在蓄电池中。

对太阳能辐射强度进行建模 ${ }^{[13]}$,太阳垂直照射 强度如下:

$$
\begin{aligned}
I_{0} & =I\left(\frac{1+\varepsilon \cos \alpha_{s}}{1-\varepsilon^{2}}\right)^{2} \\
\alpha_{s} & =2 \pi\left(n_{\mathrm{d}}-4\right) / 365
\end{aligned}
$$

式中: $I$ 为太阳常数, 取 $1367 \mathrm{~W} / \mathrm{m}^{2} ; \varepsilon$ 为地球偏心 率, 取 $0.0177 ; n_{\mathrm{d}}$ 为一年中距离 1 月 1 日的天数。太 阳辐射强度功率建模如下, 首先确定当地日 - 地连 线矢量 $n_{\mathrm{s}}$ 的方向

$$
\begin{aligned}
& \sin \beta_{\mathrm{s}}=\sin \theta_{\mathrm{s}} \sin \phi_{\mathrm{s}}+\cos \theta_{\mathrm{s}} \cos \phi_{\mathrm{s}} \cos \left(\omega_{\mathrm{s}}\right) \\
& \sin \gamma_{\mathrm{s}}=\frac{\sin \beta_{\mathrm{s}} \sin \theta_{\mathrm{s}}-\sin \phi_{\mathrm{s}}}{\cos \beta_{\mathrm{s}} \cos \theta_{\mathrm{s}}} \\
& \phi_{\mathrm{s}}=23.45 \pi \sin \left(2 \pi \times \frac{284+n_{\mathrm{d}}}{365}\right) / 180 \\
& \omega_{\mathrm{s}}=\pi-\pi t_{\mathrm{missinn}} / 12 \\
& n_{\mathrm{s}}=\left(\cos \beta_{\mathrm{s}} \sin \gamma_{\mathrm{s}}, \cos \beta_{\mathrm{s}} \cos \gamma_{\mathrm{s}}, \sin \beta_{\mathrm{s}}\right)
\end{aligned}
$$

式中: $\beta_{\mathrm{s}}$ 为太阳高度角; $\gamma_{\mathrm{s}}$ 为太阳方位角, 以正东为 0 , 向南取正; $\phi_{\mathrm{s}}$ 为赤纬角; $\theta_{\mathrm{s}}$ 为地理纬度; $\omega_{\mathrm{s}}$ 为太阳 时角; $t_{\text {mission }}$ 为一天中的时刻。太阳能辐射吸收效率 和机翼平面的法向量 $\boldsymbol{n}_{\mathrm{w}}$ 与日地连线向量夹角 $\boldsymbol{n}_{\mathrm{s}}$ 相 关, 根据几何关系, 有太阳能原始功率 $P_{\text {sun0 }}$

$$
P_{\text {sun } 0}=I_{0} \cos \left\langle\boldsymbol{n}_{\mathrm{s}}, \boldsymbol{n}_{\mathrm{w}}\right\rangle
$$

太阳能通过动力系统转化为机械能的功率 $P_{\text {sun }}$ 为

$$
P_{\text {sun }}=P_{\text {sun } 0} S_{\mathrm{w}} \eta_{\mathrm{sc}} \eta_{\text {asc }} \eta_{\mathrm{m}} \eta_{\mathrm{P}}
$$

式中: $S_{\mathrm{w}}$ 为机翼面积; $\eta_{\mathrm{sc}}$ 为光伏电池转化效率; $\eta_{\mathrm{asc}}$ 为光伏电池铺设面积与机翼面积的比值; $\eta_{\mathrm{m}}$ 为能源 管理系统效率; $\eta_{\mathrm{P}}$ 为推进系统效率。从公式中可以 看出, 在同一次飞行中, 想要让太阳能吸收能力提 高, 就应尽可能减小 $\boldsymbol{n}_{\mathrm{w}}$ 和 $\boldsymbol{n}_{\mathrm{s}}$ 之间的夹角。

\section{2 风场建模}

动态滑翔从侧向梯度风场中获取额外能量。侧 向梯度风场广泛存在于大气中, 以水面及地面上方 数十米的大气中最为典型, 处于小型无人机的主要 应用范围内。反映侧向梯度风场常用的幂数模 
型 $^{[3]}$ 表示如下

$$
V_{\mathrm{W}}(h)=V_{\mathrm{R}}\left(\frac{h}{H_{\mathrm{R}}}\right)^{p}
$$

式中: $H_{\mathrm{R}}$ 与 $V_{\mathrm{R}}$ 则指风场中某一代表高度与该高度 下对应的代表风速; $V_{\mathrm{W}}$ 指风场中随不同高度 $h$ 变化 对应的侧向风速。指数 $p$ 被称为风场强度变化指 数, 表征梯度风场的变化强度, 在不同的环境下取值 不同。不同 $p$ 值在 $H_{\mathrm{R}}=20 \mathrm{~m}, V_{\mathrm{R}}=10 \mathrm{~m} / \mathrm{s}$ 时对风场 的影响如图 1 所示。

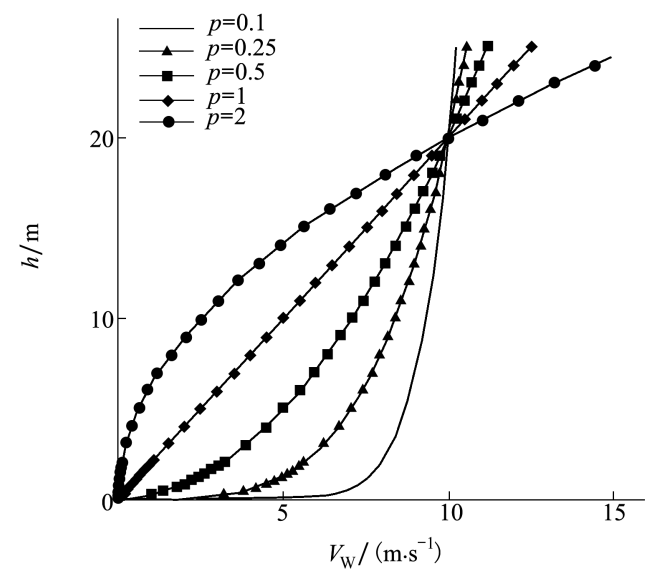

图 1 不同 $p$ 值下的梯度风场模型

在飞行仿真过程中, 假设风场风速方向沿地轴 系 $+X$ 轴方向, 即文中所有风速 $V_{\mathrm{W}}$ 均以 $+X$ 轴方向 为正方向。根据相关文献 [4], 并综合本文研究对 象应用范围, 最终选取风场强度变化指数 $p=0.25$, 风场代表高度 $H_{\mathrm{R}}=20 \mathrm{~m}, V_{\mathrm{R}}=8 \mathrm{~m} / \mathrm{s}$ 。

\section{3 动态滑翔动力学建模}

动态滑翔中飞行动力学模型采用的主要轴系及 角的定义如图 2 所示:

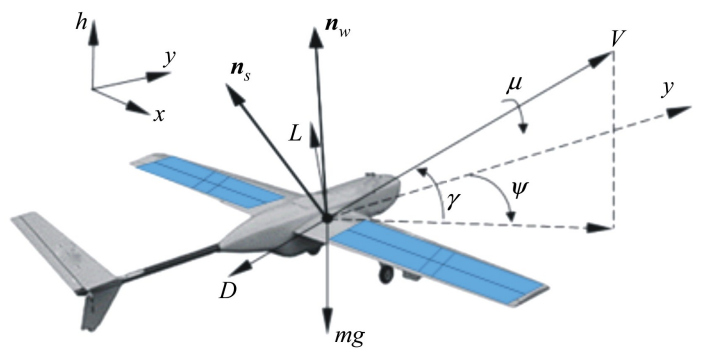

图 2 动力学模型角度及坐标定义

该模型在梯度风场中的飞行器动力学及运动学 模型可表达为

$$
\begin{aligned}
& m \dot{V}=T-D-m g \sin \gamma-m \dot{V}_{\mathrm{W}} \cos \gamma \sin \psi \\
& m V \cos \gamma \dot{\psi}=L \sin \mu-m \dot{V}_{\mathrm{W}} \cos \psi \\
& m V \dot{\gamma}=L \cos \mu+m \dot{V}_{\mathrm{W}} \sin \gamma \sin \psi-m g \cos \gamma \\
& \dot{h}=V \sin \gamma \\
& \dot{x}=V \cos \gamma \sin \gamma+V_{\mathrm{W}} \\
& \dot{y}=V \cos \gamma \cos \psi
\end{aligned}
$$

该质心动力学方程定义在航迹坐标系上。其 中, $\psi$ 为飞行器航迹偏角, $\gamma$ 为航迹倾角, $\mu$ 为航迹滚 转角; $V$ 为飞行器相对地面速度, $V_{\mathrm{W}}$ 为对应高度下风 速, 定义方向沿地轴系 $+X$ 方向; $L, D, T$ 分别为升 力、阻力与推力, 为简化计算推力方向假设和阻力方 向一致, 均定义在风轴系中, 与图 2 保持一致; $(x, y$, $h$ ) 为飞行器在地轴系下的飞行坐标。

升力与阻力计算采用简化模型如下

$$
\begin{aligned}
& L=\rho S_{\mathrm{w}} C_{\mathrm{L}} V^{2} / 2 \\
& D=\rho S_{\mathrm{w}} C_{\mathrm{D}} V^{2} / 2 \\
& C_{\mathrm{D}}=C_{\mathrm{D} 0}+K_{\mathrm{D}} C_{\mathrm{L}}^{2}
\end{aligned}
$$

式中, $C_{\mathrm{L}}$ 和 $C_{\mathrm{D}}$ 分别是升力与阻力系数, $C_{\mathrm{D} 0}$ 为零升 阻力系数, $K_{\mathrm{D}}$ 为诱导阻力因子。

动态滑翔数值仿真研究中常用的模型之一为 Deittert 等提出的小型无人机模型 ${ }^{[3-4]}$, 本文对其进 行少许修改并将来自于文献 [13] 的太阳能动力能 源系统与之结合, 选用修改后的模型进行计算。新 模型主要参数如下:

\section{表 1 无人机模型基准参数}

\begin{tabular}{cc}
\hline 参数名称 & 模型参数 \\
\hline 质量 $m / \mathrm{kg}$ & 5.443 \\
翼展 $b / \mathrm{m}$ & 4.32 \\
机翼面积 $S_{\mathrm{w}} / \mathrm{m}^{2}$ & 0.957 \\
零升阻力系数 $C_{\mathrm{D} 0}$ & 0.017 \\
诱导阻力因子 $K_{\mathrm{D}}$ & 0.0192 \\
展弦比 $\mathrm{AR}$ & 19.54 \\
最大升力系数 $C_{\mathrm{Lmax}}$ & 1 \\
最大升阻比 $(L / D)_{\max }$ & 28 \\
最大滚转角 $\left.\mu_{\max } /{ }^{\circ}\right)$ & 60 \\
光伏电池光电转化效率 $\eta_{\mathrm{sc}}$ & 0.2 \\
光伏电池铺设面积比 $\eta_{\mathrm{asc}}$ & 0.8 \\
能源管理系统效率 $\eta_{\mathrm{m}}$ & 0.95 \\
推进系统效率 $\left(\right.$ 含电机与桨) $\eta_{\mathrm{P}}$ & 0.72 \\
\hline
\end{tabular}




\section{2 能量方程}

\section{1 能量变化分析}

应用动态滑翔技术的小型太阳能飞行器飞行过 程中主要的能量获取来自经由动态滑翔从风场中获 取的能量 $E_{\text {wind }}$ 和经由太阳能动力能源系统获得的 太阳能 $E_{\mathrm{sun}}$; 主要的能量支出来自空气阻力带来的 能量损失 $E_{\mathrm{D}}$ 和动力系统做功消耗的能量 $E_{\mathrm{T}}$, 其中 $E_{\mathrm{T}}$ 消耗的能量会补充飞行器的机械能。

飞行器的机械能可以表示为

$$
E_{\text {mechnical }}=m g h+\frac{1}{2} m V^{2}
$$

式中, $E_{\text {mechnical }}$ 为机械能。小型无人机在整个飞行过 程中认为质量是恒定的, 保持不变, 因此, 能量相对 时间的变化率表示为

$$
\dot{E}_{\text {mechnical }}=m g\left(\dot{h}+\frac{1}{g} V \dot{V}\right)
$$

同时,引人辅助公式

$$
\begin{gathered}
\dot{h}=V_{\sin \gamma} \\
\dot{V}_{\mathrm{W}}=\frac{\mathrm{d} V_{\mathrm{W}}}{\mathrm{d} h} \dot{h}
\end{gathered}
$$

将公式(6)、(10) 和(11) 代人(9) 式进行整理 可以得到

$$
\dot{E}_{\text {mechnical }}=-\frac{\mathrm{d} V_{\mathrm{W}}}{\mathrm{d} h} m V^{2} \sin \gamma \cos \gamma \sin \psi+T V-D V
$$

(12) 式反映了飞行器飞行过程中的能量变化 规律, 式中的 3 项分别代表经由动态滑翔从风场中 获取能量、动力系统做功、飞行过程中气动阻力消耗 的能量。合理调整航迹倾角 $\gamma$ 与航迹偏角 $\psi$ 使得 $\sin \gamma \sin \psi<0$, 从而使得第一项为正, 即飞行器从风 场中获取能量, 即在 $\mathrm{d} V_{\mathrm{W}} / \mathrm{d} h>0$ 的风场中, 飞行器 动态滑翔获能的主要飞行策略应为逆风爬升后顺风 下降。因此,通过合理的飞行控制、姿态控制及航迹 规划, 能够使得飞行器在不损失机械能的条件下完 成飞行,进而提升航程或航时。

太阳能动力能源系统的能量变化规律为通过太 阳能摄人能量, 通过动力系统输出能量, 为保证和机 械能变化量的可对比性, 分析中采用动力能源系统 输出端能量值。该能量计算如下

$$
E_{\text {sun_power }}=E_{\text {sun }}-E_{\mathrm{T}}
$$

在进行周期性盘旋飞行的过程中, 每个周期持 续的时间相对于地球自转时间短得多, 可以近似认

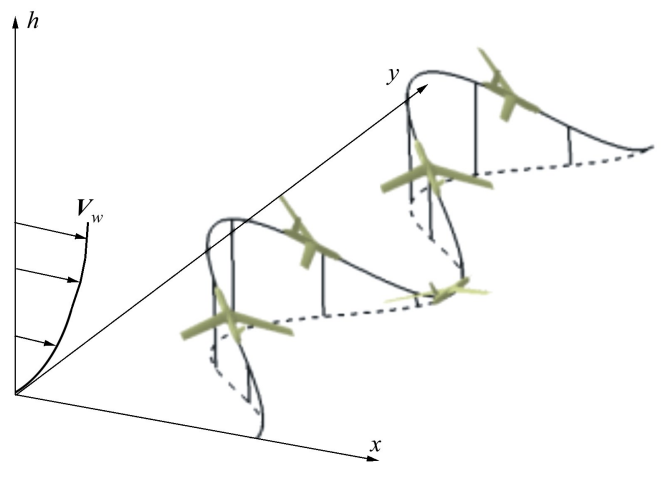

图 3 动态滑翔示意图

为对单个周期进行能量分析过程中 $\boldsymbol{n}_{\mathrm{s}}$ 的方向和太 阳垂直照射强度 $I_{0}$ 保持不变, 因此太阳能动力能源 系统的能量的变化率主要取决于机翼法向量, 当机 翼法向量与太阳人射向量 $\boldsymbol{n}_{\mathrm{s}}$ 夹角越小时, 整体太阳 能获能能力越强

$$
\begin{gathered}
\dot{E}_{\text {sun } \_ \text {power }}=P_{\text {sun }}\left(\cos \left\langle\boldsymbol{n}_{\mathrm{w}}, \boldsymbol{n}_{\mathrm{s}}\right\rangle\right)-T V \\
n_{\mathrm{w}}=\left(\begin{array}{l}
\sin \mu \cos \psi-\sin \gamma \cos \mu \sin \psi, \\
-\sin \mu \sin \psi-\sin \gamma \cos \mu \cos \psi, \\
\cos \mu \cos \gamma
\end{array}\right)^{\mathrm{T}}
\end{gathered}
$$

因此, 为提高太阳能获取, 飞行中的姿态会使得 机翼法向量更趋向于太阳人射方向, 最终使飞行轨 迹所围面的中点法线更趋向于 $\boldsymbol{n}_{\mathrm{s}}$ 方向。

从能量总体收支功率角度看, 飞行过程中, 主要 的能量收人为动态滑翔风场吸收能量功率 $P_{\text {wind }}$ 和 太阳能能量收人功率 $P_{\text {sun }}$, 主要能量支出为飞行阻 力消耗功率 $P_{\mathrm{D}}$ 和动力系统能量消耗功率 $P_{\mathrm{T}}$ 。衡量 总体能量收支变化率的最终表达式为

$$
\begin{gathered}
\Delta \dot{E}=\left(E_{\text {wind }}+E_{\text {sun }}-E_{\mathrm{T}}-E_{\mathrm{D}}\right)^{\prime}= \\
P_{\text {wind }}+P_{\text {sun }}-P_{\mathrm{T}}-P_{\mathrm{D}}= \\
-\frac{\mathrm{d} V_{\mathrm{W}}}{\mathrm{d} h} m V^{2} \sin \gamma \cos \gamma \sin \psi+ \\
P_{\text {sun }}\left(n_{\mathrm{w}}\right)-T V-D V
\end{gathered}
$$

\section{2 最优能量获取方式分析}

通过能量方程的分析, 确定了结合 2 种技术的 小型无人机飞行过程中主要的能量获取来自动态滑 翔从梯度风场中获取的风能 $E_{\text {wind }}$ 和通过太阳能动

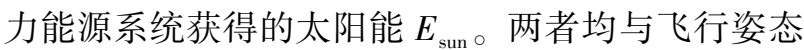
有关, 通过合理调整飞行器的飞行姿态, 可以最大化 能量获取功率。

动态滑翔风场吸收能量功率 $P_{\text {wind }}$ 表达式为 


$$
\begin{gathered}
P_{\text {wind }}=-\frac{\mathrm{d} V_{\mathrm{W}}}{\mathrm{d} h} m V^{2} \sin \gamma \cos \gamma \sin \psi= \\
-\frac{1}{2} \frac{\mathrm{d} V_{\mathrm{W}}}{\mathrm{d} h} m V^{2} \sin 2 \gamma \sin \psi
\end{gathered}
$$

分析 $P_{\text {wind }}$ 表达式可以发现, 风能吸收效率与航 迹倾角和航迹偏角有关, 逆风爬升和顺风俯冲过程 中 $P_{\text {wind }}$ 为正, 能够有效吸收风场能量, 反之则支出 能量。当 $\psi=90^{\circ}, \gamma=-45^{\circ}$ 或 $\psi=-90^{\circ}, \gamma=45^{\circ}$ 时, 即 飞行器沿顺风航向以 $45^{\circ}$ 角向下俯冲或沿逆风方向 以 $45^{\circ}$ 角向上爬升时, 飞行器能够最大化获取梯度 风场能量

$$
\max \left(P_{\text {wind }}\right)=\frac{1}{2} \frac{\mathrm{d} V_{W}}{\mathrm{~d} h} m V^{2}
$$

此时, 风场能量获取功率与风梯度 $\mathrm{d} V_{W} / \mathrm{d} h$ 与飞 行速度有关。根据小型无人机实际飞行可能的环 境, 取 $\mathrm{d} V_{W} / \mathrm{d} h=0.2 \mathrm{~s}^{-1}, V=21 \mathrm{~m} / \mathrm{s}$ 时, 风场能量获 取功率与飞行姿态的关系如图 4 所示。

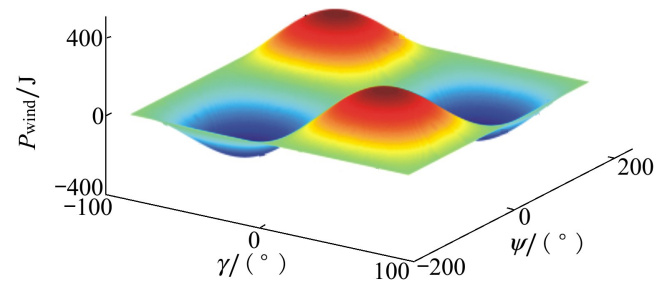

图 4 风场能量获取功率与飞行姿态的关系
从图中可以看出, 风场能量获取有 2 个最大值, 分别对应前文分析的 2 种情况, 同时也有 2 个最小 值, 分别为逆风俯冲和顺风爬升点, 符合前文对 $P_{\text {wind }}$ 的分析。

太阳能能量获取功率 $P_{\text {sun }}$ 表达式为

$$
\begin{aligned}
& P_{\text {sun }}=I_{1} \cos \left\langle\boldsymbol{n}_{\mathrm{s}}, \boldsymbol{n}_{\mathrm{w}}\right\rangle \\
& I_{1}=I_{0} S_{\mathrm{w}} \eta_{\text {sc }} \eta_{\text {asc }} \eta_{\mathrm{m}} \eta_{\mathrm{P}}
\end{aligned}
$$

式中

$$
\begin{gathered}
\boldsymbol{n}_{\mathrm{s}}=\left(\cos \beta_{\mathrm{s}} \sin \gamma_{\mathrm{s}}, \cos \beta_{\mathrm{s}} \cos \gamma_{\mathrm{s}}, \sin \beta_{\mathrm{s}}\right)=\left(n_{\mathrm{s} 1}, n_{\mathrm{s} 2}, n_{\mathrm{s} 3}\right) \\
\boldsymbol{n}_{\mathrm{w}}=\left(\begin{array}{l}
\sin \mu \cos \psi-\sin \gamma \cos \mu \sin \psi, \\
-\sin \mu \sin \psi-\sin \gamma \cos \mu \cos \psi, \\
\cos \mu \cos \gamma
\end{array}\right)^{\mathrm{T}} \quad(20) \\
\text { 公式 }(19) \text { 中 } \cos \left\langle\boldsymbol{n}_{\mathrm{s}}, \boldsymbol{n}_{\mathrm{w}}\right\rangle \text { 可以展开为 } \\
\cos \left\langle\boldsymbol{n}_{\mathrm{s}}, \boldsymbol{n}_{\mathrm{w}}\right\rangle=(\sin \mu \cos \psi-\sin \gamma \cos \mu \sin \psi) n_{\mathrm{s} 1}+ \\
(-\sin \mu \sin \psi-\sin \gamma \cos \mu \cos \psi) n_{\mathrm{s} 2}+\cos \mu \cos \gamma n_{\mathrm{s} 3}= \\
\left(\cos \psi n_{\mathrm{s} 1}-\sin \psi n_{\mathrm{s} 2}\right) \sin \mu+ \\
\left(-\sin \gamma \sin \psi n_{\mathrm{s} 1}-\sin \gamma \cos \psi n_{\mathrm{s} 2}+\cos \gamma n_{\mathrm{s} 3}\right) \cos \mu= \\
A \sin \mu+B \cos \mu \\
\quad \text { 若公式中滚转角是一个自由变量, 当滚转角取 } \\
\text { 值为 } \sin \mu=A / \sqrt{A^{2}+B^{2}} \text { 时, } \cos \left\langle\boldsymbol{n}_{\mathrm{s}}, \boldsymbol{n}_{\mathrm{w}}\right\rangle \text { 取得最大 } \\
\text { 值, 如公式 }(22) \text { 所示: }
\end{gathered}
$$

$$
\max \left(\cos \left\langle\boldsymbol{n}_{\mathrm{s}}, \boldsymbol{n}_{\mathrm{w}}\right\rangle\right)=\sqrt{A^{2}+B^{2}}=\sqrt{\left(\cos \psi n_{s 1}-\sin \psi n_{s 2}\right)^{2}+\left(-\sin \gamma\left(\sin \psi n_{s 1}+\cos \psi n_{s 2}\right)+\cos \gamma n_{s 3}\right)^{2}}
$$

选取飞行日期 $n_{\mathrm{d}}=180$, 飞行时刻 $t$ 为 12 时 为例, 取 $I_{1}=553.8 \mathrm{~J}$, 太阳能获取效率 $P_{\text {sun }}$ 与飞行姿 态之间的关系如图 5 所示。

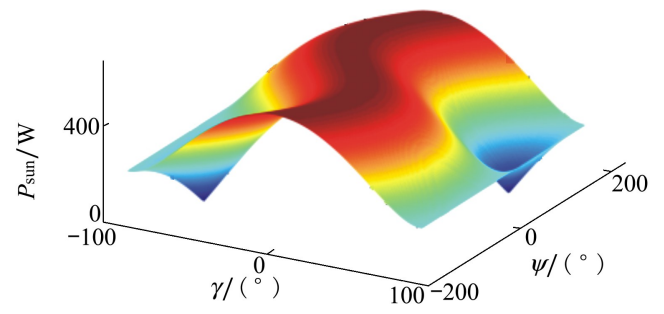

图 5 太阳能获取效率与飞行姿态的关系

图 5 中可以看到,能取得最大值的为一系列点, 反映了此时航迹倾角与航迹偏角之间的取值联系, 此时如公式(23) 取 


$$
\begin{gathered}
P_{\mathrm{in}}=-\frac{\mathrm{d} V_{\mathrm{W}}}{\mathrm{d} h} m V^{2} \sin \gamma \cos \gamma \sin \psi+ \\
I_{1} \sqrt{\left(\cos \psi n_{\mathrm{s} 1}-\sin \psi n_{\mathrm{s} 2}\right)^{2}+\left(-\sin \gamma\left(\sin \psi n_{\mathrm{s} 1}+\cos \psi n_{\mathrm{s} 2}\right)+\cos \gamma n_{\mathrm{s} 3}\right)^{2}}
\end{gathered}
$$

根据小型无人机实际飞行可能的环境, 选取 $\mathrm{d} V_{W} / \mathrm{d} h=0.2 \mathrm{~s}^{-1}, V=21 \mathrm{~m} / \mathrm{s}, n_{\mathrm{d}}=180, t_{\text {mission }}$ 为 12 时, $I_{1}=553.8 \mathrm{~J}$ 时, 能量吸收功率与飞行姿态之 间的关系如图 6 所示:

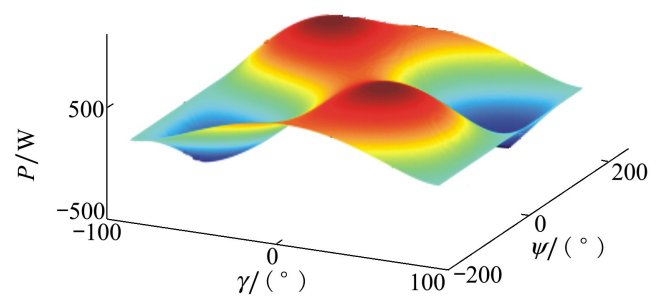

图 6 能量吸收功率与飞行姿态的关系

该状态在不限制滚转角的前提下, 能量吸收的 最大值处更接近动态滑翔风场获能最大值处, 且偏 向太阳能获取最大值点方向。主要原因在于两者取 最大值的点较为接近, 而太阳能吸收功率最大值附 近梯度较小。

在一天中的不同时刻, $\boldsymbol{n}_{\mathrm{s}}=\left(n_{\mathrm{s} 1}, n_{\mathrm{s} 2}, n_{\mathrm{s} 3}\right)$ 取值 不同,也会影响能量吸收功率。作上述环境下 $t_{\text {mission }}$ 分别取 6 时、9 时、15 时、18 时的总能量吸收功率与 飞行姿态关系图, 可以与图 6 中 12 时结果进行 对比:

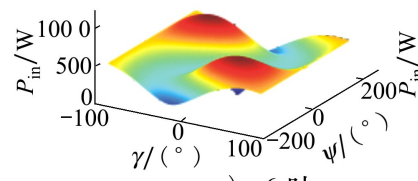

a) 6 时

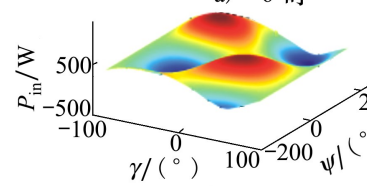

c) 15 时

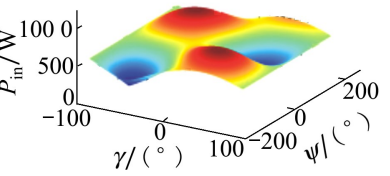

b) 9 时

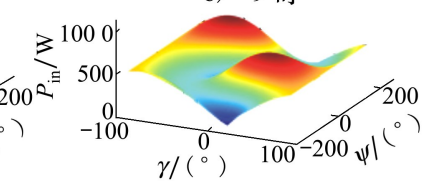

d) 18 时
图 7 一天中的不同时刻能量吸收功率与飞行姿态关系图

可以看到, 飞行时刻不同对能量获取功率有一 定影响, 但最值仍集中在动态滑翔风场获能最大值 附近, 偏向太阳能获取最大值方向。实际飞行过程 中考虑飞行轨迹中的约束与控制要求, 各姿态变量 不一定能够满足最大能量获取要求, 但最优姿态仍 然是在满足逆风爬升与顺风俯冲条件下, 尽可能减
小太阳人射向量与机翼平面法向量间的夹角。

\section{3 仿真分析}

为了验证结合太阳能与动态滑翔技术的飞行器 能量变化规律, 将飞行过程中的能量变化直观展现 出来, 从而得到 2 种技术结合的能量获取优势的结 论, 本文根据表 1 中典型飞行器的参数, 搭建了仿真 系统, 优化工具选择 SNOPT, 求解软件为 MATLAB, 通过最优化理论进行了仿真计算, 获得了最佳能量 获取的控制和轨迹曲线, 并分析了飞行中的能量变 化。优化目标为最大能量获取

$$
\min J=-\int_{0}^{t_{n}} \Delta \dot{E} \mathrm{~d} t
$$

结合太阳能与动态滑翔飞行动力学模型表示 如下

$$
\dot{\boldsymbol{x}}(t)=f(\boldsymbol{x}(t), \boldsymbol{u}(t))
$$

式中: $\boldsymbol{x}=[V, \psi, \gamma, h, x, y]$ 为系统的状态向量; $\boldsymbol{u}=$ $\left[\mu, C_{\mathrm{L}}, T\right]$ 为输人控制向量。飞行轨迹离散为 $M$ 个 点, 每个点的状态向量可以由初始状态和飞行控制 决定, 因此设计变量为

$$
\begin{aligned}
& \boldsymbol{X}=\left[x_{1,1}, \cdots, x_{6,1}, u_{1,1}, u_{2,1}, \cdots,\right. \\
& \left.u_{1, M}, u_{2, M}, t_{f}\right]= \\
& \quad\left[\boldsymbol{x}_{1}^{\mathrm{T}}, \boldsymbol{u}_{1}^{\mathrm{T}}, \cdots, \boldsymbol{u}_{M}^{\mathrm{T}}, t_{f}\right] \\
& x_{i, m+1}=x_{i, m}+ \\
& \quad\left(k_{i, m}^{1}+2 k_{i, m}^{2}+2 k_{i, m}^{3}+k_{i, m}^{4}\right) \Delta t / 6
\end{aligned}
$$

选取周期性环绕航迹作为目标优化路径。飞行 器在完成一周期循环后应能返回初始飞行状态, 以 保证该循环的可重复性。根据这一条件, 得到约束 如下

$$
\begin{aligned}
& V\left(t_{f}\right)=V\left(t_{0}\right) \\
& \lambda\left(t_{f}\right)=\lambda\left(t_{0}\right) \\
& h\left(t_{f}\right)=h\left(t_{0}\right) \\
& \psi\left(t_{f}\right)=\psi\left(t_{0}\right) \\
& x\left(t_{f}\right)=x\left(t_{0}\right) \\
& y\left(t_{f}\right)=y\left(t_{0}\right)
\end{aligned}
$$

为保证飞行器飞行安全, 在飞行过程中还应避 免飞行器及其机翼触地或没人水中, 转化为高度约 
束有

$$
h-\frac{1}{2} b|\sin (\mu)| \geqslant h_{\text {min }}
$$

飞行器模型采用飞行仿真中较为常用的带动力 太阳能飞行器模型,其基本参数如表 2 所示。

表 2 方法验证优化模型初始参数设置和环境参数

\begin{tabular}{cc}
\hline 初始参数名称 & 初值设置 \\
\hline 初始速度 $V_{1} /\left(\mathrm{m} \cdot \mathrm{s}^{-1}\right)$ & 18 \\
初始航迹偏角 $\psi_{1} /\left(^{\circ}\right)$ & 0 \\
初始航迹倾角 $\gamma_{1} /\left(^{\circ}\right)$ & 0 \\
初始坐标 $\left(x_{1}, y_{1}, h_{1}\right) / \mathrm{m}$ & $(0,0,12)$ \\
轨迹离散点个数 $M$ & 100 \\
飞行时长初值 $t_{f 0} / \mathrm{s}$ & 9 \\
代表风速值 $V_{\mathrm{R}} /\left(\mathrm{m} \cdot \mathrm{s}^{-1}\right)$ & 8 \\
地理纬度 $\theta_{\mathrm{s}} /\left({ }^{\circ}\right)$ & 45 \\
飞行时刻 $t_{\mathrm{mission}}$ & $18: 00$ \\
飞行日期 $n_{\mathrm{d}} / \mathrm{h}$ & 180 \\
\hline
\end{tabular}

表 2 给出了优化初始参数设置。优化过程中, 各输人参数还需要满足飞行动力学模型的约束, 如 升力和滚转角控制量需要满足 $C_{\mathrm{Lmax}}$ 和 $\mu_{\text {max }}$ 的限制。 同时选取夏季黄昏时间作为飞行的背景环境, 此时 单纯太阳能不足以完全支撑飞行能量的损失, 选择 此时刻以方便讨论太阳能和动态滑翔能量获取过程 中的变化规律。仿真飞行路径结果如图 8 所示。

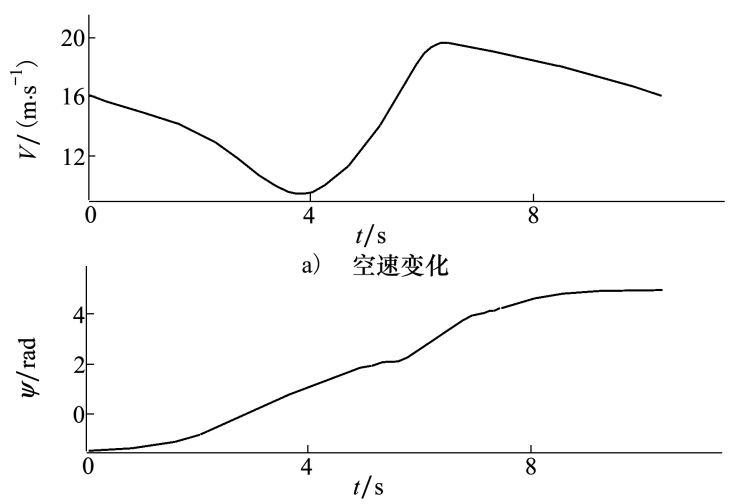

b）航迹偏角变化

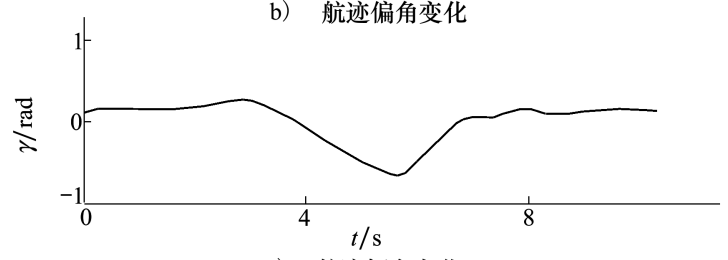

c）航迹倾角变化

图 9 空速、航迹偏角、航迹倾角随时间的变化图

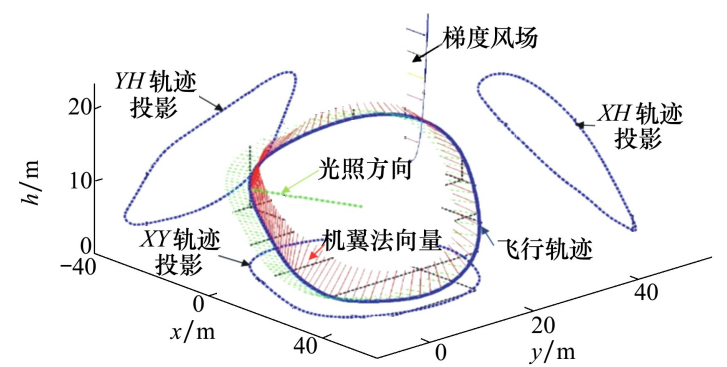

图 8 仿真飞行路径

图 8 中, 实线为飞行器仿真飞行轨迹, 虚线为飞 行轨迹在 3 个主平面上的投影, 点划线为太阳人射 向量, 同时在图中标注了飞行器在路径各点处的机 翼平面法线向量。图中还展示了实际使用的风场形 状。从图中可以看出, 经过路径的规划, 飞行器轨迹 基本满足逆风爬升、顺风俯冲的动态滑翔获能基本 原则, 同时路径的主要法线方向尽可能朝向日照向 量方向, 满足最大获取太阳能的需求。飞行过程中 的飞行速度、航迹偏角和航迹倾角如图 9 所示。通 过空速变化结合环绕路径, 可以看到飞行速度和飞 行高度在完成一个飞行周期后均与初值保持不变, 总机械能在周期前后变化量基本为 0 ,即飞行器在 不损失机械能的条件下完成了一个周期的环绕飞 行,执行了监视任务。对应的控制变量如图 10 所示。
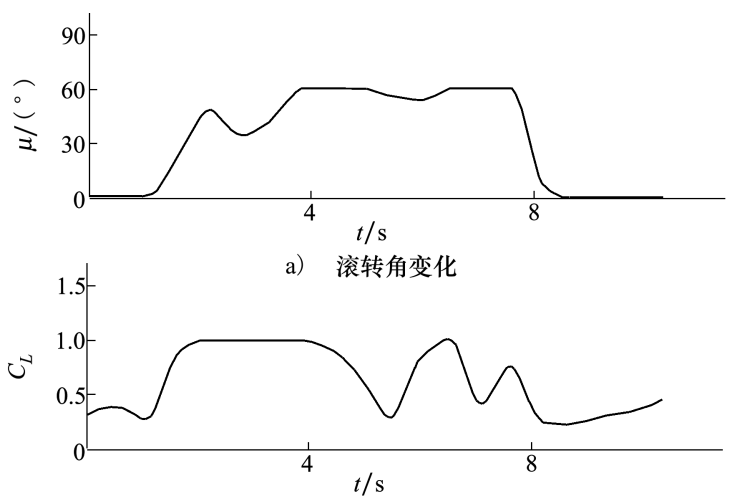

b) 升力系数变化

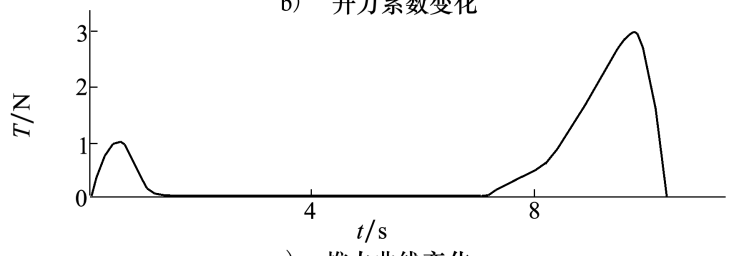

c) 推力曲线变化

图 10 滚转角、升力系数、推力曲线随时间的变化图 
飞行器机械能及其相关能量变化项如图 11 所示。

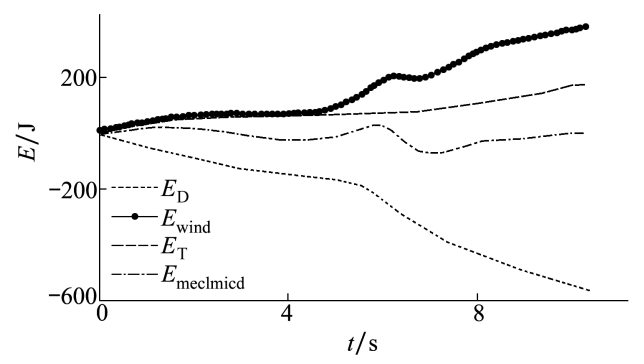

图 11 飞行器机械能及其相关能量变化项变化

图中可以看到,机械能在周期完成后变化量与 初值相同,其中通过动态滑翔吸收的风场能量是主 要的机械能能量补充来源, 在能量补充中占主导地 位。通过对比空速变化图可以发现, 动态滑翔能量 吸收处于平台段对应速度的最低点 $(t \approx 4 \mathrm{~s})$ 和最高 点 $(t \approx 6 \mathrm{~s})$, 即对应航迹的最高点与最低点, 其余点 处飞行器基本满足逆风爬升和顺风俯冲机动, 动态 滑翔吸收能量得到增长。航迹最低点 $(t \approx 6 \mathrm{~s})$ 后, 飞行器主要处于逆风爬升段, 此段中空气阻力消耗 能量增加, 同时动力系统做功也需要增加以完成最 终周期闭环, 补充了部分机械能。通过机械能变化 分析, 可以确定动态滑翔技术确实能够从风场中获 取额外能量,进而提升飞行器的续航能力。
总体能量收支变化如图 12 所示, 仿真飞行轨迹与传 统太阳能飞行器等效盘旋轨迹 (等效投影面积, 等 效飞行速度, 因此可能飞行周期时间不同, 两者之间 能量对比时采用平均功率对比) 以及无阳光照射纯 动态滑翔飞行的对比航迹如图 13 所示, 三者能量平 均功率变化如表 3 所示。

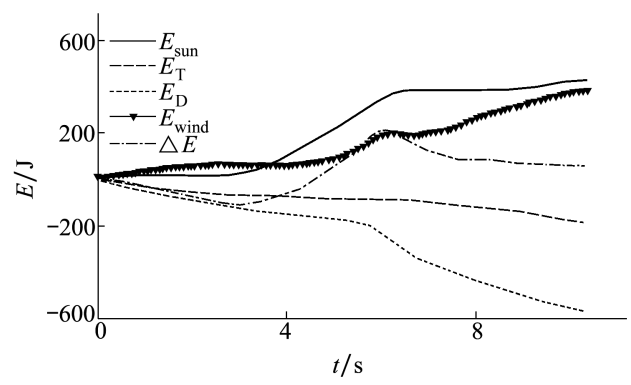

图 12 总体能量收支变化

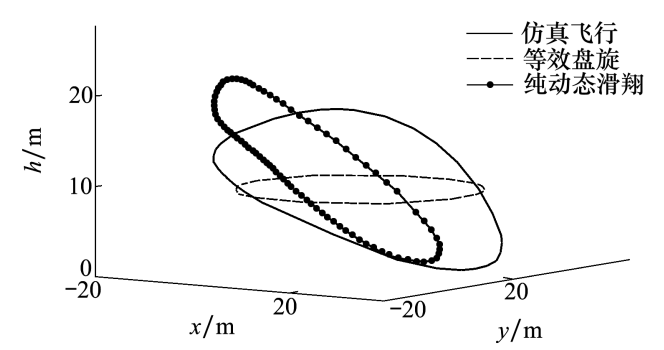

(opt 为仿真飞行轨迹, circle 为等效盘旋, DS 为纯动态滑翔轨迹)

图 13 仿真飞行轨迹与其等效盘旋轨迹

表 3 动态滑翔飞行与其等效盘旋能量平均功率变化

$\mathrm{J} / \mathrm{s}$

\begin{tabular}{cccc}
\hline 平均功率 & 仿真飞行 & 等效盘旋 & 纯动态滑翔 \\
\hline 动态滑翔风场吸收能量平均功率 & 37.76 & 0 & 50.86 \\
太阳能能量收人平均功率 & 42.26 & 29.73 & 0 \\
飞行阻力消耗能量平均功率 & 55.42 & 46.41 & 54.60 \\
动力系统能量消耗平均功率 & 17.67 & 46.41 & 3.87 \\
机械能变化平均功率 & 0.02 & 0 & 0.12 \\
总体能量收支变化平均功率 & 6.93 & -63.09 & -7.61
\end{tabular}

通过对比结果可以看出, 在飞行时刻 (夏季的 $18: 00$, 接近日落) 太阳能通过规划仍有足够的能量 吸收量, 在抵消动力系统及阻力能量损耗的同时, 仍 有 $6.93 \mathrm{~J} / \mathrm{s}$ 的能量吸收功率, 蓄电池仍处于充电状 态, 传统的盘旋飞行方式则需要平均每秒支出 $63.09 \mathrm{~J}$, 纯动态滑翔飞行能量支出功率为 $7.61 \mathrm{~J} / \mathrm{s}$, 这部分能量需要由动力能源系统的蓄电池提供。对
比仿真结果和纯动态滑翔的结果, 可以看到为了顾 及太阳能的能量获取, 动态滑翔能量摄人比纯动态 滑翔飞行稍小, 但结合 2 种方法的总能量收入功率 为 $80.03 \mathrm{~J} / \mathrm{s}$, 比纯动态滑翔高 $36.5 \%$, 比传统盘旋纯 太阳能飞行能量收人高 $62.8 \%$ 。仿真整体飞行轨迹 的法线方向则更偏向于日照的方向, 这是为了获取 更多太阳能同时兼顾动态滑翔获能而做出的妥协。 
从各方法总体能量收支变化平均功率对比结果可以 看出, 结合太阳能和动态滑翔技术的飞行器从风场 和太阳能中获取了更多额外能量, 能够支持更长时 间的飞行, 能够有效提升飞行器的续航能力。

\section{4 结 论}

1) 本文从能量角度分析了用太阳能技术与动 态滑翔技术的飞行器飞行过程中的能量变化原理, 分别探讨了机械能、太阳能动力能源系统以及全机 总体能量收支变化的基本原理与各能量分项, 提出 了表征结合 2 种技术的飞行器的能量收支公式, 以 及太阳能和风场能量最大获取的基本飞行策略与控 制策略。
2) 通过能量公式的推导与分析, 确定了动态滑 翔获取风能的基本飞行策略为逆风爬升与顺风俯 冲, 太阳能能量获取最佳策略在于尽可能使飞行中 机翼平面法线方向与光线人射方向夹角减小。

3）建立了基于梯度风场的小型无人机飞行动 力学模型与相应的太阳能动力能源系统模型, 并依 照能量分析中得到的能量收支公式与获能飞行控制 策略进行了仿真验证, 验证了在日落时分环绕飞行 的飞行器通过合适的飞行控制策略能够有效获得足 够的额外能量支持飞行, 相较于传统的盘旋飞行和 纯动态滑翔飞行能量收人功率分别提高 $36.5 \%$ 和 $62.8 \%$,证明了结合 2 种获能技术能够有效提升飞 行器的续航性能, 为今后进一步研究全天候不间断 飞行提供了基础。

\section{参考文献:}

[1] SAChS G, TRAUgOTT J, NeSterova A P, et al. Flying at no Mechanical Energy Cost: Disclosing the Secret of Wandering Albatrosses[J]. Plos One, 2012, 7(9) : 41449

[2] ZHAO Y. Optimal Patterns of Glider Dynamic Soaring $[\mathrm{J}]$. Optimal Control Application and Methods, 2004, 25: 67-89

[3] SACHS G, BUSSOTTI P. Application of Optimal Control Theory to Dynamic Soaring of Seabirds [J]. Variational Analysis and Applications, 2005, 79: 975-994

[4] DeitTert M, Richards A, TOOmer C A, et al. Engineless Unmanned Aerial Vehicle Propulsion by Dynamic Soaring[J]. Journal of Guidance, Control, and Dynamics, 2009, 32(5): 1446-1457

[5] 朱炳杰, 侯中喜. 无人机风梯度滑翔过程中能量变化 $[\mathrm{J}]$. 国防科技大学学报, 2015, 37( 1) : 78-83

ZHU Bingjie, HOU Zhongxi. Energy Transformation in Dynamic Soaring of Unmanned Aerial Vehicles [J]. Journal of National University of Defense Technology, 2015, 37(1) : 78-83 (in Chinese)

[6] LIU D N, HOU Z X, GUO Z, et al. Optimal Patterns of Dynamic Soaring with a Small Unmanned Aerial Vehicle[J]. Journal of Aerospace Engineering, 2017, 231(9):1593-1608

[7] 单上求, 侯中喜, 朱炳杰, 等. 动态滑翔动力学建模与风梯度能量获取 [ $\mathrm{J}]$. 国防科技大学学报, 2015(4) :45-49 SHAN Shangqiu, HOU Zhongxi, ZHU Bingjie, et al. Dynamics Modeling of Dynamic Soaring and Energy Gaining from the Wind Gradient $[\mathrm{J}]$. Journal of National University of Defense Technology, 2015(4) :45-49 (in Chinese)

[8] SPANGELO S C, GILBERT E G. Power Optimization of Solar-Powered Aircraft with Specified Closed Ground Tracks [J]. Journal of Aircraft, 2013, 50(1): 232-238

[9] SINEGLAZOV V M, KARABETSKY D P. Energy System Design of Solar Aircraft[C] // 2013 IEEE 2nd International Conference on Actual Problems of Unmanned Air Vehicles Developments, 2013

[10] SINEGLAZOV V M, KARABETSKY D P. Flying wing Design for Solar Rechargeable Aircraft[C] // 2013 IEEE 2nd International Conference on Actual Problems of Unmanned Air Vehicles Developments, 2013

[11] 昌敏, 周洲, 郑志成. 太阳能飞机原理及总体参数敏度分析 $[\mathrm{J}]$. 西北工业大学学报, 2010, 28(5): 792-796 CHANG Min, ZHOU Zhou, ZHENG Zhicheng. Flight Principles of Solar-Powered Airplaneand Sensitivity Analysis of Its Conceptual Parameter[J]. Journal of Northwestern Polytechnical University, 2010, 28(5): 792-796 (in Chinese)

[12] 昌敏, 周洲, 李盈盈. 基于能量平衡的太阳能飞机可持续高度分析 $[\mathrm{J}]$. 西北工业大学学报, 2012, 30(4) : 541-546 CHANG Min, ZHOU Zhou, LI Yingying. An Effective Theoretical Analysis of Persistent Flight Altitudes of Solar-Powered Airplanes [J]. Journal of Northwestern Polytechnical University, 2012, 30(4) : 541-546 (in Chinese)

[13] 马东立, 包文卓, 乔宇航. 基于重力储能的太阳能飞机飞行轨迹研究 [J]. 航空学报, 2014, 35(2):408-416 
MA Dongli, BAO Wenzhuo, QIAO Yuhang. Study of Flight Path for Solar-powered Aircraft Based on Gravity Energy Reservation [J]. Acta Aeronautica et Astronautica Sinica, 2014, 35(2):408-416 (in Chinese)

\title{
Analysis of Flight Energy Variation of Small Solar UAVs Using Dynamic Soaring Technology
}

\author{
LIU Siqi, BAI Junqiang \\ (School of Aeronautics, Northwestern Polytechnical University, Xi'an 710072,China)
}

\begin{abstract}
Limited by its low energy capacity, small UAVs have short flight time. Therefore, it is of great significance to improve the endurance performance of small UAVs by applying solar energy technology and dynamic soaring technology which can obtain external energy. This paper analyzes the energy exchange principle of small UAVs using solar energy technology and dynamic soaring technology, and works out the strategy of maximum energy gain. Small UAV flight dynamics model combined with solar power-energy system was built, choosing surround flight path at twilight moment for simulation validation. The advantages of small solar UAV using dynamic soaring technology in energy acquisition in hovering flight and pure dynamic gliding flight are verified, compared with traditional solar vehicle. The significance of combining the two energy acquisition technologies to improve the endurance of small UAV is proved, which provides a basis for further study of all-weather uninterrupted flight in the future.
\end{abstract}

Keywords : dynamic soaring; small UAV ; trajectory optimization; solar energy ; energy analysis 